\section{Superconducting magnets}

No longer are superconducting magnets confined to research laboratories or those which use nuclear magnetic resonance (NMR) machines for more routine measurements. With the development of techniques that, for example, permit the use of NMR in the study of living tissues, it is natural that the body-scanning fraternity should have devised machines intended to provide biochemically based whole-body scans of human beings. This development entails the construction of magnets providing a uniform magnetic field of 2 tesla or so over regions $100 \mathrm{~cm}$ in dimension - for practical purposes unattainable without the use of superconducting magnets.

One of the British companies active in the field, Oxford Instruments Limited, has already built a number of magnets of this type. Physiological whole-body scanning is for the time being directed at two objectives - the use of the resonances of ${ }^{31} \mathrm{P}$ as indicators of physiological function,

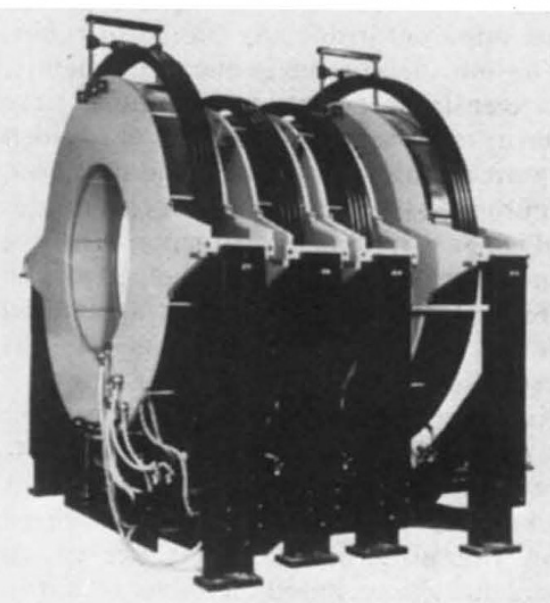

A 0.15-tesla four-coil resistive magnet for whole-bod imaging. Clear-bore access is $800 \mathrm{~mm}$.

and of proton resonances as indicators of where water is more plentiful in the body (which could be an alternative to the use of diagnostic $\mathrm{X}$ rays).

Whether large-volume magnetic fields for such purposes will prove to be in great demand will no doubt hang on the outcome of the several clinical studies with prototype machines now being planned. The magnet manufacturers will be overjoyed if it turns out that scanning procedures yielding representations of the structure of cross-sections through a human body (such as may be constructed from suitable proton magnetic resonance measurements) are preferable to the more familiar use of diagnostic $X$ rays. Meanwhile, the supply of large-volume magnets for the prototype machines, at a cost of $£ 100,000$ or so each, seems to be a sufficient recompense for the high cost of development.

In the evolution of superconducting magnets for laboratory use, the cutting edge of development seems to be what it has been for the past twenty years - the improvement of magnets for use in nuclear magnetic resonance. For the higher the value of the magnetic field in which a sample is immersed, the greater the resolution that will ultimately be possible. But the sharpness of the spectral lines that eventually emerge will also be determined by the uniformity of the field over the volume of whatever sample is being used, with the result that stronger fields must also be more uniform.

Standard superconducting magnets with pre-specified field homogeneity can now be bought off the shelf. Central field strengths of these standard products range up to 14 tesla (140,000 gauss), but the magnets themselves may be no more than a foot long. The compactness of the range of superconducting magnets now available is perhaps their most striking characteristic, especially when the imagination is invited to speculate about the bulk of the power supply equipment that would be necessary to generate fields of a tesla (the maximum obtainable with conventional electromagnets).

\section{Cooling problems}

The analogous penalty in the use of laboratory superconducting magnetics is, however, the cryostatic equipment needed to maintain the superconductor at liquid helium temperatures. The coils of a superconducting magnet must be immersed in liquid helium or, alternatively, in liquid-helium vapour. Naturally enough, users of superconducting magnets have no wish to double as low-temperature physicists, worrying about the adequacy of the cooling system. Fortunately, most of the suppliers of laboratory magnets have been able to reduce the rate at which helium is lost from their cryostats to the point at which topping up the system with liquid helium is necessary only at intervals of a few months. The ideal is that replenishment can be tied in with routine maintenance visits.

For the rest, the design of a super- conducting magnet raises few problems not encountered by the traditional designers of electromagnets. The simplest magnets are solenoids. Often it is convenient to split the solenoids in half, on a plane perpendicular to the axis, but at the expense of maximum field strength. Improving the homogeneity of the field, increasing the volume over which irregularities do not exceed some specified percentage, is to some extent a function of the way the coils are wound, but the inclusion of shims of superconducting material is usually also advantageous.

Quite apart from considerations of compactness, the overrriding attraction of superconducting magnets over conventional electromagnets is that the maximum field is not limited by the magnetic permeability of the core material, soft iron perhaps. Yet there are limits to the extent to which the field strength of a superconducting magnet can be increased which are themselves determined by the properties of the superconductors used for constructing the coils. The crucial consideration is that the material of which the coils are made should not experience magnetic fields great enough to rob them of the superconducting properties. With existing superconducting alloys (niobiumtitanium and niobium-tin) it should be possible to push beyond 17 tesla, the present limit of what is practicable.

The most straightforward way of using this material is to deposit it on layers of a tape of copper and stainless steel (relatively an insulator at liquid helium temperatures, but a means of carrying current if superconducting properties should at any point be lost). More recently, techniques have been developed for forming niobiumtin alloy from filaments of niobium which are annealed with bronze in such a way that the tin component of the bronze preferentially alloys with the niobium. The residual copper serves both as a mechanical support and as a means of conduction should superconducting properties be lost.

Where the design of superconducting magnets will go from here is anybody's guess. The few companies specializing in laboratory-scale magnets (beam-forming

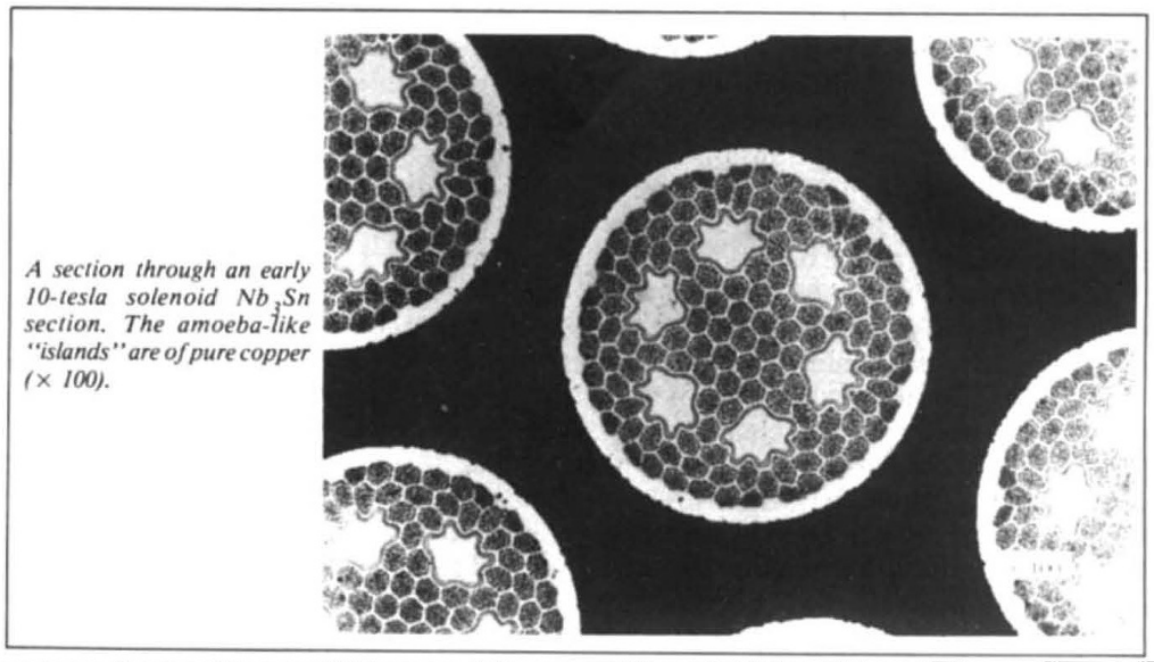


magnets are another business) usually of fer custom-built designs intended to suit the needs of people in academic or industrial laboratories in need of some predetermined configuration of magnetic field. As with NMR, from any one of these applications might spring some new range of off-the-shelf superconducting magnets.

Oxford Instruments, one of the principal suppliers, says that it will consider ad hoc proposals from potential academic customers, but will not in the first instance produce a detailed estimate of the cost of building a new design. Rather, it will undertake to produce information sufficient to sustain a research grant application, and will produce a final estimate of cost in return for a fee refundable against a firm order when the application has been successful.

Whether this esoteric business will - or would be - transformed by the development of superconducting materials with transition temperatures substantially above the boiling point of liquid helium is another matter. The chief benefit, as things are, would be quantitative: helium consumption would be reduced. But if somebody were to construct a material superconducting at liquid nitrogen temperatures, a whole new ballpark would be accessible...

\section{High-energy physics rides on flux}

THERE is a real sense in which the development of high-energy physics in the past half century has been sustained by the technology of the magnet builders. Although the first important landmark in the artificial manipulation of nuclear structure - the use of $300 \mathrm{MeV}$ protons for the disintegration of nuclei by Cockcroft and Walton in 1931 - was made possible by means of an electrostatic accelerator, E.O. Lawrence was even then building the first cyclotron at Berkeley. By the time, in 1935 or so, that the limits of electrostatic acceleration had been reached, working cyclotrons were all the rage.

The principle of these machines is simple enough. The path of a charged particle travelling in a magnetic field will be curved, but no kinetic energy will be lost. If the magnetic field is sufficiently extensive, the particle will travel in a closed path, thus increasing the length of time for which other means of accelerating it can do their work.

The cyclotrons which in the 1930s followed the Berkeley design were the simplest of all accelerating machines. The principle is simply to construct a substantial volume of uniform magnetic field between the poles of an electromagnet, to provide a source of charged particles at the centre of the arrangement and to arrange for some means of adding to the kinetic energy of the charged particles as they traverse closed orbits between the poles of the source magnet.

Cyclotrons have functioned since the

\section{Cold comfort at room temperature}

SUPERCONDUCTIVITY is the next best thing to perpetual motion: an electrical current once established in a superconducting circuit will keep going indefinitely. So is it not mere misfortune that the only circumstances in which known materials can be made superconducting are those involving temperatures in the liquid helium range, below $4.1 \mathrm{~K}$ or thereabouts?

After the best part of a decade, the hopes first fostered by the empirical discovery that suitably constructed linear polymer materials have electronic conduction bands and are indeed capable of conducting electricity might make possible the design of molecules exhibiting superconductivity at room temperatures. In reality, painful experience has shown that superconductivity is doggedly a low-temperature phenomenon, involving lattice or molecular vibrations (phonons) and electron movement in a cooperative transition from a disordered to an ordered state. Room-temperature superconductivity is likely always to lie in the future.

For practical purposes, it is a sufficient but still daunting goal to find materials superconducting at the temperature of liquid nitrogen, the common outer refrigerant in superconducting cooling systems.

The record so far is a transition temperature of $21 \mathrm{~K}$ for a version of $\mathrm{Nb}_{3} \mathrm{Ge}$ in which germanium atoms are partly replaced by atoms of aluminium. (This is a true superconductor of Type II, able to sustain high magnetic fields without losing its essential properties, but metallurgically hard to work with.) As things are, such interest in the search for improved superconductors centres on the sulphides of molybdenum doped with rare-earth elements as substitutes for $\mathrm{Mb}$.

In practice, even unexpectedly rapid progress in the search for superconductors with higher transition temperatures would only slowly transform the technology of making superconducting magnets. In practice the improved superconductors now coming into use are metallurgically almost impossible. They function as planned only if pure and stoichiometrically exact. It will be some time before it is a matter of routine to wind kilometres of wire on some form and call the result a magnet.

the Second World War) is a formidable undertaking.

For one thing, the sheer bulk of the early 1930s because the frequency with which a charged particle traverses its closed orbit in a magnetic field is independent of the dimensions of the orbit and simply a function of the characteristics of the particle and the strength of the field $(B)$. Thus the frequency of rotation is given by the familiar relationship $e B / 2 \pi m$, where $e$ and $m$ are the charge and mass of the particles.

Since the beginning, cyclotrons have been no better and no worse than the design of their magnets. Since the first machine at Berkeley, the standard procedure has been to accelerate charged particles by means of alternating electrostatic fields within a pair of semicircular hollow conducting surfaces shaped so that, when put together, they would constitute a hollow pancake-shaped structure - and which, when separate, are know as "dees". The two halves of this structure are fed with radiofrequency power at the characteristic frequency of the cyclotron (a function only of $B$ and the particle concerned).

One obvious difficulty with which the magnet designers have had since the beginning to contend is the sheer physical problem of shaping the magnetic field appropriately. Since charged particles spiral outwards from the central point at which they are injected, the field must be for practical purposes uniform over the maximal orbits followed by the particles. The construction of electromagnets spanning close on $2 \mathrm{~m}$ (such as with the 184-inch cyclotron built at Berkeley after magnetic circuit required to provide a return path for the magnetic field between the poles of the electromagnet where the action is is almost a feat of mechanical engineering in its own right. For another, the shaping of the pole pieces (usually machined from cast or forged blocks of soft iron) is a complicated process of balancing the loss of flux through the sides of tapering circular endpieces against the need for uniformity of field.

Throughout the 1930s, the construction of cyclotron magnets was almost a black art. Once a machine had been built, the designers of the magnet would be required to fiddle around with the placing of shims near the poles of the magnets to improve the characteristics of the field, its uniformity in particular. Even the first cyclotrons, however, were blessed with one important built-in benefit - because, between the poles of an electromagnet, flux lines unavoidably become more distant from each other, displacements of circulating charged particles towards one pole or another are discouraged by the mirroring effect thereby produced.

For practical purposes, cyclotrons have been used for accelerating protons and heavier ions, with maximum energy (determined by the area of the space between the pole pieces) measured in tens of $\mathrm{MeV}$. The acceleration of electrons to comparable and greater energies is best accomplished by means of linear accelerators (most spectacularly by the two-mile accelerator at Stanford, 